\title{
Open-Lake Experimental Investigation of Azimuth Angle Estimation Using a Single Acoustic Vector Sensor
}

\author{
Anbang Zhao, ${ }^{1,2,3}$ Lin Ma, ${ }^{1,2}$ Juan Hui $\mathbb{D}^{1,2}$ Caigao Zeng, ${ }^{1,2}$ and Xuejie Bi ${ }^{1,2}$ \\ ${ }^{1}$ College of Underwater Acoustic Engineering, Harbin 150001, China \\ ${ }^{2}$ Science and Technology on Underwater Acoustic Laboratory, Harbin 150001, China \\ ${ }^{3}$ National Key Laboratory of Science and Technology on Underwater Acoustic Antagonizing, China State Shipbuilding Corporation \\ Systems Engineering Research Institute, Beijing 100036, China
}

Correspondence should be addressed to Juan Hui; huijuan_heu@126.com

Received 22 July 2017; Revised 10 November 2017; Accepted 5 February 2018; Published 27 March 2018

Academic Editor: Jaime Lloret

Copyright (c) 2018 Anbang Zhao et al. This is an open access article distributed under the Creative Commons Attribution License, which permits unrestricted use, distribution, and reproduction in any medium, provided the original work is properly cited.

\begin{abstract}
Five well-known azimuth angle estimation methods using a single acoustic vector sensor (AVS) are investigated in open-lake experiments. A single AVS can measure both the acoustic pressure and acoustic particle velocity at a signal point in space and output multichannel signals. The azimuth angle of one source can be estimated by using a single AVS in a passive sonar system. Open-lake experiments are carried out to evaluate how these different techniques perform in estimating azimuth angle of a source. The AVS that was applied in these open-lake experiments is a two-dimensional accelerometer structure sensor. It consists of two identical uniaxial velocity sensors in orthogonal orientations, plus a pressure sensor-all in spatial collocation. These experimental results indicate that all these methods can effectively realize the azimuth angle estimation using only one AVS. The results presented in this paper reveal that AVS can be applied in a wider range of application in distributed underwater acoustic systems for passive detection, localization, classification, and so on.
\end{abstract}

\section{Introduction}

Research on underwater acoustic systems has been receiving an increasing attention in both military and civilian applications [1-4]. Compared with acoustic pressure sensors (a.k.a. scalar hydrophone), acoustic vector sensors (AVS) can simultaneously measure the acoustic pressure along with acoustic particle velocity at a signal point in space $[1,3]$. Better detection effectiveness and higher estimation precision can be achieved by fully making use of the pressure and the additional particle velocity information [5]. For several decades, AVSs have been ubiquitously applied in source direction of arrival (DOA) estimation and localization [6,7]. In recent years, AVSs have been increasingly extended to multiple research fields, such as feature extraction of underwater target signal [8], underwater target tracking [1], underwater acoustic communication $[9,10]$, acoustic focusing and shielding $[3,11]$, and geoacoustic inversion problem [12]. Therefore, experimental investigation of the AVS signal processing methods is very important in practical engineering applications.

It is well known that the AVS array processing could greatly enhance the performance of detection and estimation [13-16]. AVS arrays are able to estimate both elevation and azimuth without left-right ambiguity and give better result compared with scalar sensor. However, as demand for smaller arrays has significantly increased, the large-scale aperture of traditional sensor arrays restricts their practical application fields. Consequently, single-transmit singlereceive (SISO) distributed sonar system, such as sonobuoys and other small-scale detection equipment, is very popular in distributed detection systems for their own characteristics of layout convenience, high stealthiness [17], and so on The DOA estimation of the underwater source could be realized by using only one single AVS. Thus, the single AVS passive sonar detection system and the AVS signal processing research attract ever-increasing attention.

A sizable literature exists on the methods for processing signals of a single AVS $[5,6,17-23]$. Researchers put forward 
a method for DOA estimation based on average acoustic intensity processing. This method is experimentally investigated in [18]. Another proposed azimuth angle estimation algorithm based on complex acoustic intensity measurement is experimentally investigated in [19]. These results show that the method is able to effectively detect the underwater source and estimate its azimuth angle. Furthermore, researchers also propose an improved method based on complex acoustic intensity weighted statistics and another method aiming to detect a target that radiating line spectra. The method is experimentally verified in [17]. This approach based on complex acoustic intensity weighted statistics can suppress broadband coherent interference. Methods described in [17-19] are all based on acoustic intensity measurement. The azimuth angle is estimated by directly computing the acoustic intensity component of the different coordinate axis.

As mentioned earlier, a single AVS measures a Cartesian component of the acoustic particle velocity vector of the incident wavefield and simultaneously outputs multichannel signals. Hence, array signal processing methods can be applied by regarding the AVS as an array. A twodimensional AVS has $3 \times 1$ array manifold, in response to a unit-power underwater acoustic wave front [1]. In [18, 19], the conventional beamforming approach to estimating DOA based on a single AVS is evaluated by experiments. Reference [6] introduces a new underwater acoustic eigenstructure ESPRIT-based algorithm that yields closed-form DOA estimates using a single AVS. The minimum variance distortionless response (MVDR) beam patterns associated with a single AVS distant from any reflecting boundary is characterized in [20]. Most of the direction-finding (DF) methods using an AVS are based on the far-field measurement model. Missing in the literature is a near-field measurement model for the AVSs. In [21], a measurement model is presented there for an AVS located near a reflecting boundary. Taking into account the characteristics of one single AVS, the highresolution DOA estimation based on time-space transform and subspace decomposing theory is realized in [22]. Moreover, [23] investigates how the AVS's DF accuracy would be degraded by random deviations from its nominal gain response and/or phase response.

Even though extensive researches on AVS processing have been done in the past thirty years, studies focusing on the experimental investigation are rarely presented. In order to motivate practical engineering application demands in passive sonar detection system, we study five well-known azimuth angle estimation methods using a single AVS and carry out a comprehensive experimental research of these methods.

The five noted methods, namely, complex acoustic intensity measurement (CAIM) method, weighted complex acoustic intensity measurement (WCAIM) method, conventional beamforming $(\mathrm{CBF})$ method, minimum variance distortionless response (MVDR) beamforming method, and multiple signal characteristic (MUSIC) method, are investigated in this paper. The first two methods are based on complex acoustic intensity measurement. Compared with other methods based on acoustic intensity measurement, they do

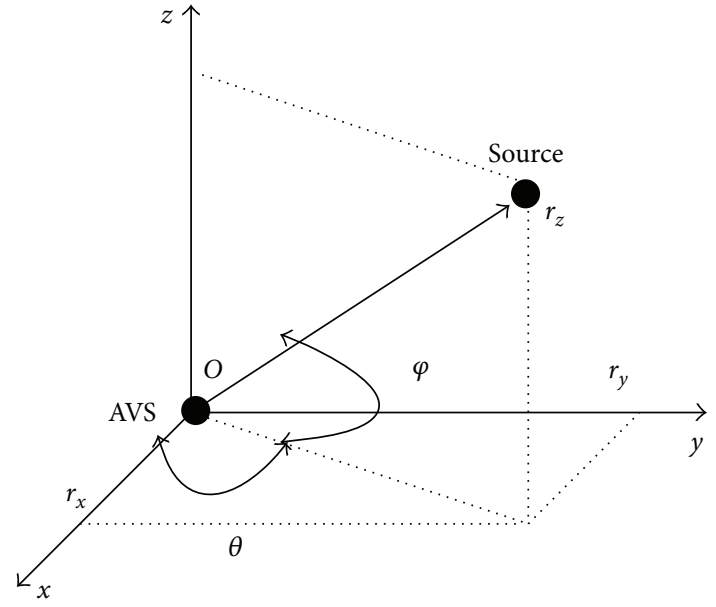

Figure 1: AVS coordinates and the propagation of planar wave front with azimuth angle $\theta$ and elevation angle $\varphi$. (Figure reproduced from Zhao et al. [17], under the Creative Commons Attribution License/public domain).

well in the condition of either in line-spectra coherent interference lying in wideband signal or in wideband coherent interference lying in a line-spectra signal. The rest three methods investigated are mainly applied in an array that consists of many sensors. The same as [18-23], these methods can be extendedly applied by regarding the AVS as an array. $\mathrm{CBF}$ is the most traditional and the most classic technique used to estimate azimuth angle of an acoustic source. MVDR aims to let the signal of interest be received without any distortion while minimizing the noise arriving from other directions. MUSIC is one of the most popular algorithms capable of super-resolution performance. MUSIC method requires knowledge of a number of sources to perform accurate subspace decomposition of the covariance matrix.

The main contribution of this paper is the experimental investigation of five azimuth angle estimation methods upon a single vector-sensor configuration. In this paper, our attention will be restricted to the case of one single underwater acoustic source. The rest of this paper is organized as follows: Section 2 describes the theoretical background. The AVS measurement model and the five investigated methods are briefly reviewed. Section 3 consists of open-lake experiments, data processing results, and estimation performance discussion. Finally, conclusions are totally drawn in Section 4.

\section{Measurement Model and Methods}

2.1. AVS Model. From now on, and without loss of generality, an acoustic wave is assumed to be propagating in a quiescent, homogeneous, and isotropic fluid in this paper. In addition, it is explicitly assumed that the impinging signals are plane waves. The plane-wave acoustic pressure at a frequency $\omega$ can be given as $[18,19]$

$$
p(r, t)=p_{0} e^{j\left(\mathbf{k}^{T} \cdot \mathbf{r}-\omega t\right)},
$$




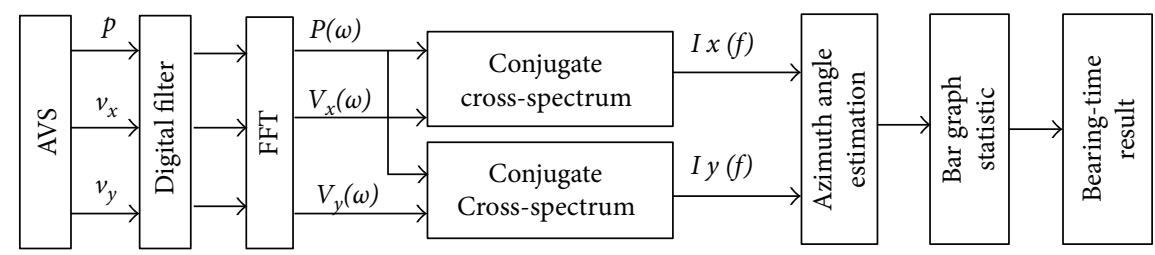

Figure 2: Illustration of CAIM and WCAIM. (Figure reproduced from Zhao et al. [17], under the Creative Commons Attribution License/ public domain).

where $\omega$ denotes the angular frequency, $\mathbf{k}$ symbolizes the wavenumber vector, and $\mathbf{r}$ represents the space vector. Figure 1 represents the AVS coordinates, when a source signal impinges upon the sensors from an azimuth angle $\theta$ and an elevation angle $\varphi$.

The wavenumber vector $\mathbf{k}$ has the form

$$
\mathbf{k}=\left[\begin{array}{c}
k_{x} \\
k_{y} \\
k_{z}
\end{array}\right]=k\left[\begin{array}{c}
\cos \theta \cos \varphi \\
\sin \theta \cos \varphi \\
\sin \varphi
\end{array}\right],
$$

where $k$ is defined as

$$
k=\sqrt{k_{x}^{2}+k_{y}^{2}+k_{z}^{2}}=\frac{\omega}{c}=\frac{2 \pi f}{c},
$$

where $c$ denotes the speed of acoustic propagation. Any point in a three-dimensional space can be commonly located by a vector $\mathbf{r}=\left[r_{x}, r_{y}, r_{z}\right]^{T}$, where $r_{x}, r_{y}$, and $r_{z}$ are the coordinates of the point in the Cartesian coordinate system. Hence, (1) has the following form:

$$
\begin{gathered}
p(r, t)=p_{0} \exp \left[j \left(k r_{x} \cos \theta \cos \varphi+k r_{y} \sin \theta \cos \varphi\right.\right. \\
\left.\left.+k r_{z} \sin \varphi-\omega t\right)\right] .
\end{gathered}
$$

And then, (4) is substituted into the equation of motion:

$$
\frac{\partial \mathbf{v}}{\partial t}+\frac{1}{\rho} \nabla p=0
$$

where $\rho$ is the medium density, and we can express (5) as

$$
\begin{aligned}
\mathbf{v} & =-\frac{1}{\rho} \int \nabla p \cdot d t, \\
\mathbf{v}(\mathbf{r}, t) & =\frac{p(\mathbf{r}, t)}{\rho c}(\cos \theta \cos \varphi \vec{\xi}+\sin \theta \cos \varphi \overrightarrow{\boldsymbol{\eta}}+\sin \varphi \vec{\zeta}),
\end{aligned}
$$

where $\zeta, \boldsymbol{\eta}$, and $\xi$, respectively, symbolize the unit vector of $x$-, $y$-, and $z$-axes. This paper will only concentrate on azimuth angle $\theta$. In this case, elevation angle $\varphi$ is assumed to be equal to zero. The acoustic pressure and acoustic particle velocity depend on time only in the measurement model. Therefore, the parameter $\mathbf{r}$ is omitted from these variables.

AVS is applied to measure the acoustic pressure component and two orthogonal components of particle velocity in the $x-O-y$ plane. Finally, the copoint output

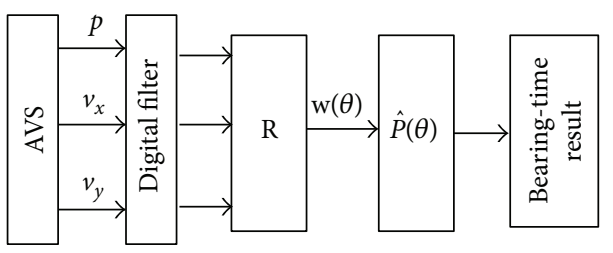

FIgUre 3: Illustration of azimuth angle estimation by CBF.

mathematical expression of the measurement model can be reduced as

$$
\begin{aligned}
p(t) & =p(t), \\
v_{x}(t) & =\frac{1}{\rho c} p(t) \cos \theta, \\
v_{y}(t) & =\frac{1}{\rho c} p(t) \sin \theta .
\end{aligned}
$$

Equation (7) indicates that the velocity direction characteristics of AVS are independent of frequency. The directivity of AVS is frequency independent as well. Consequently, a single AVS array manifold is equivalently defined in a general form as

$$
\mathbf{a}_{s}(\theta)=\left[1, \frac{1}{\rho c} \cos (\theta), \frac{1}{\rho c} \sin (\theta)\right]^{T}
$$

where $\theta \in[0,2 \pi)$ is the corresponding azimuth angle of an acoustic source measured from the positive $x$-axis and $T$ denotes the transpose. The AVS's array manifold is independent of the incident wavefield's frequency and bandwidth.

2.2. Methods. There are a variety of methods proposed by researchers to estimate the azimuth angle of the underwater acoustic source using a single AVS. In this paper, five popular methods are experimentally investigated and compared. These methods' theoretical background is briefly reviewed in the following text.

2.2.1. CAIM and WCAIM. The WCAIM method is derived from CAIM method. As shown in Figure 2 [17, 19], azimuth angle estimation by these two methods is all based on complex acoustic intensity measurements. However, they are quite different in terms of their statistical weighted value. 


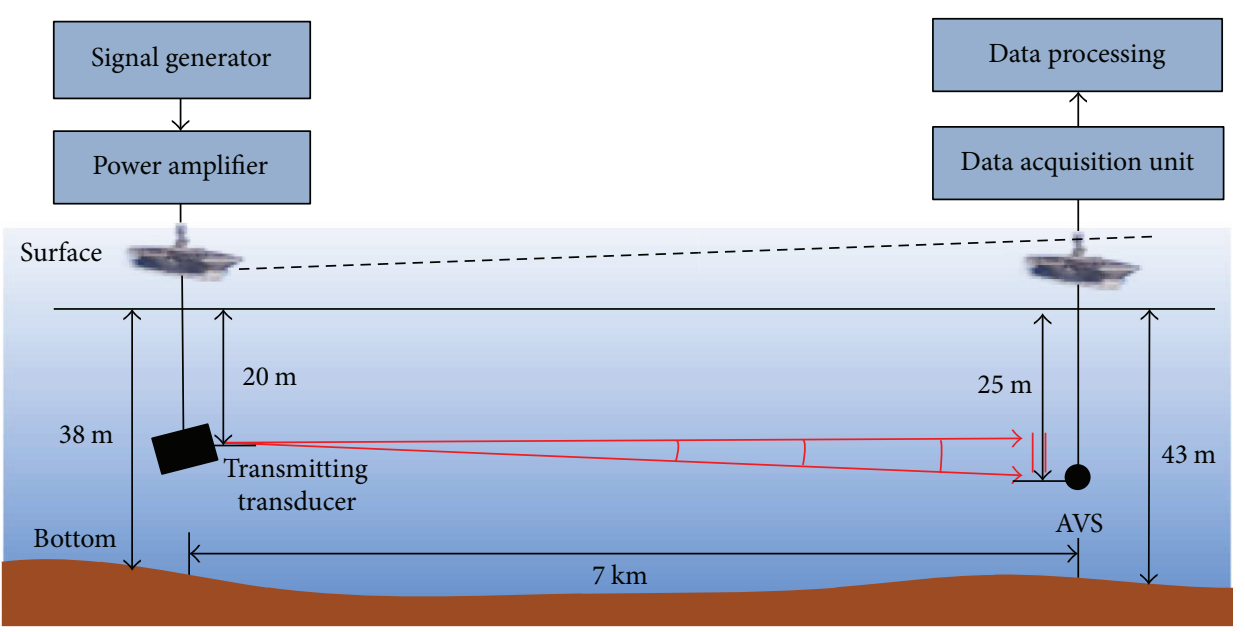

FIgURE 4: The experimental setup for azimuth angle estimation using a single AVS.

It yields frequency spectrum $P(\omega)$ and $V_{i}(\omega)(i=x, y)$ by applying fast Fourier transform (FFT) to $p(t)$ and $v_{i}(t)$ $(i=x, y)$. Hence, the acoustic pressure and velocity crossspectrum is defined as

$$
S_{p v_{i}}=P(\omega) \cdot V_{i}^{*}(\omega) \quad(i=x, y),
$$

where "*” symbolizes the complex conjugate operation. The azimuth angle of each frequency based on the acoustic pressure and velocity conjugate cross-spectrum is straightforward to calculate

$$
\theta(\omega)=\arctan \frac{I_{R_{y}}}{I_{R_{x}}}=\arctan \frac{\operatorname{Re}\left\{<P(\omega) V_{y}^{*}(\omega)>\right\}}{\operatorname{Re}\left\{<P(\omega) V_{x}^{*}(\omega)>\right\}},
$$

where "Re" represents the real part. Then, the probability density statistics can be estimated by counting the azimuth angle calculation results of all frequency points. The result is the azimuth estimation curve at a certain moment.

The typical bar graph statistics are given by

$$
\begin{aligned}
n & =\left[\theta(\omega) \times \frac{180}{\pi}\right], \\
\eta(n) & =\eta(n)+1,
\end{aligned}
$$

where $n$ is denoted as angle characterized by degree and "[]" represents getting integer operation. $\eta$ denotes a data array, which stores the frequency of each angle in $[0,360)$. The initial value of $\eta$ is set to be zero. We note that all the statistic weighted value is one in each frequency. This is the socalled CAIM method.

CAIM is improved by particularly considering the influence of the cross-spectrum energy in different frequency point as $[17,19]$

$$
\eta(n)=\eta(n)+W,
$$

where $W$ is the statistic weighted value of cross-spectrum. The only difference between CAIM and WCAIM is the statistic weighted value, that is, (10) and (11). The corresponding

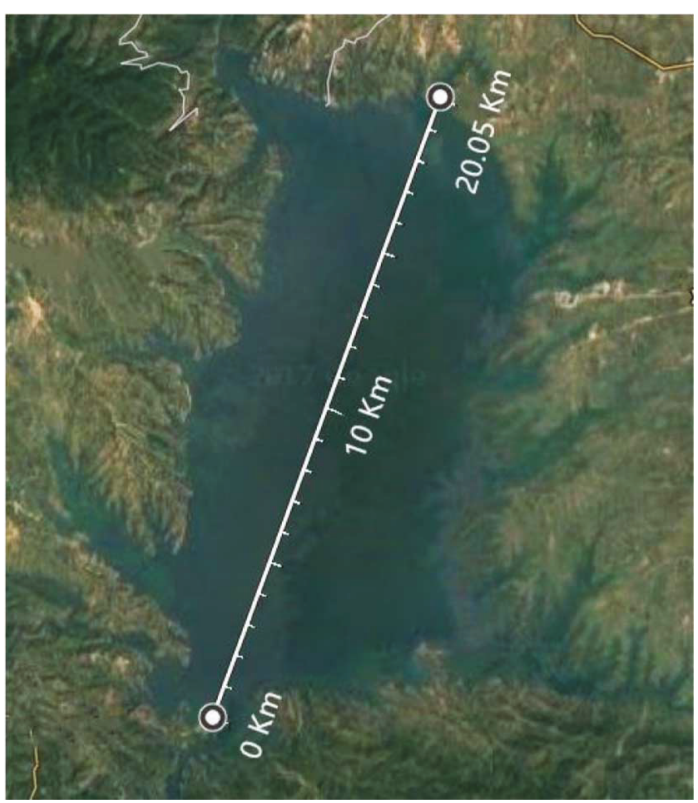

Figure 5: Map of the experiment site.

maximum value on the result curve is the source azimuth angle estimated value.

2.2.2. AVS CBF. We assume that a signal $s(t)$ is impinging upon the AVS from the angle $\theta$ and that the source is in the far field of the AVS. The vector array output $\mathbf{y}(t)$ is then $[6]$

$$
\mathbf{y}(t)=\mathbf{a}_{s}(\theta) s(t)+\mathbf{n}(t)
$$

where $\mathbf{n}(t)$ is a vector of additive noise representing the effect of undesired signals. The combined beamformer output is given by

$$
y_{c}(t)=\mathbf{w}^{H} \mathbf{y}(t)=\mathbf{w}^{H} \mathbf{a}_{s}(\theta) s(t)+\mathbf{w}^{H} \mathbf{n}(t),
$$




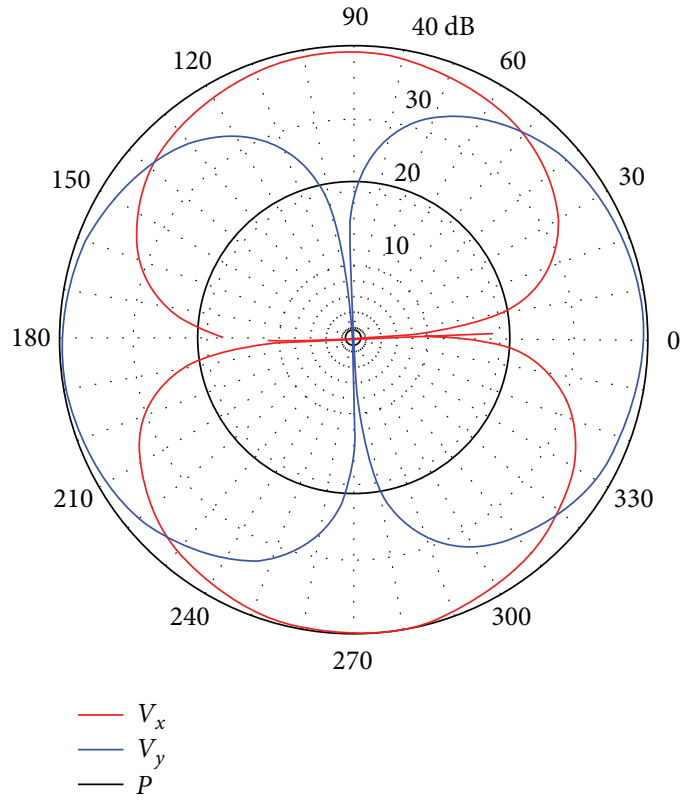

(a)

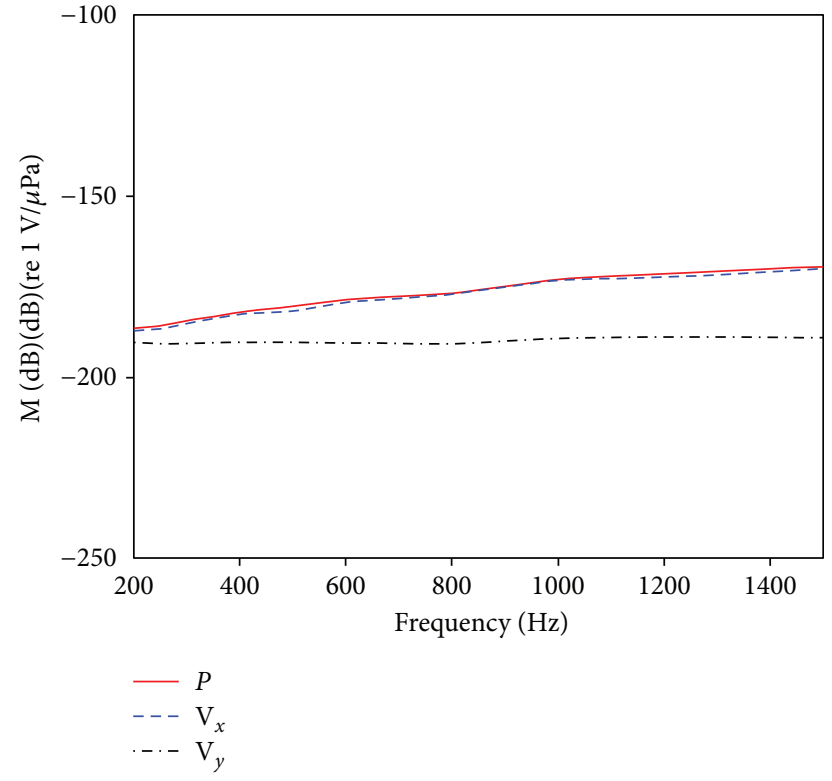

(b)

FIgURe 6: (a) The AVS directivity patterns (at $1000 \mathrm{~Hz}$ ). (b) The AVS sensitivity between $200 \mathrm{~Hz}$ and $1500 \mathrm{~Hz}$. (Figure reproduced from Zhao et al. [17], under the Creative Commons Attribution License/public domain).

where $\mathbf{w}$ is a vector of weights and " $H$ " denotes the conjugate transpose. The spatial spectra obtained by beam scanning is defined as

$$
\widehat{P}(\theta)=\left|y_{c}(t, \theta)\right|^{2}=\mathbf{w}^{H} \mathbf{R} \mathbf{w},
$$

where

$$
\mathbf{R}=E\left\{\mathbf{y} \mathbf{y}^{H}\right\}=\mathbf{a}_{s}(\theta) \mathbf{a}_{s}^{H}(\theta) E\left[s^{2}(t)\right]+E\left[\mathbf{n}(t) \mathbf{n}^{H}(t)\right],
$$

where $E\left[s^{2}(t)\right]$ and $E\left[\mathbf{n}(t) \mathbf{n}^{H}(t)\right]$ are independent of $t$. Thus, $\mathbf{R}$ is independent of $t$. In practical applications, $\mathbf{R}$ is replaced by the sample covariance matrix $\widehat{\mathbf{R}}$, where

$$
\widehat{\mathbf{R}}=\frac{1}{N} \sum_{n=1}^{N} \mathbf{y}_{n} \mathbf{y}_{n}^{H},
$$

with $N$ denoting the number of snapshots and $\mathbf{y}_{n}$ representing the $n$th snapshot.

The position corresponding to maximum value of $\widehat{P}(\theta)$ is the source azimuth angle estimated result. In Figure 3, we show an illustration of azimuth angle estimation by AVS CBF.

2.2.3. AVS MVDR. Another extensively used estimation technique is MVDR. The beamformer mainly aims to let the signal of interest be received without any distortion while minimizing the noise arriving from other directions. The design issue on weight vector can be formulated as

$$
\begin{gathered}
\min _{\mathbf{w}} \quad \mathbf{w}^{H} \mathbf{R w}, \\
\text { s.t. } \quad \mathbf{w}^{H} \mathbf{a}_{s}(\theta)=1 .
\end{gathered}
$$

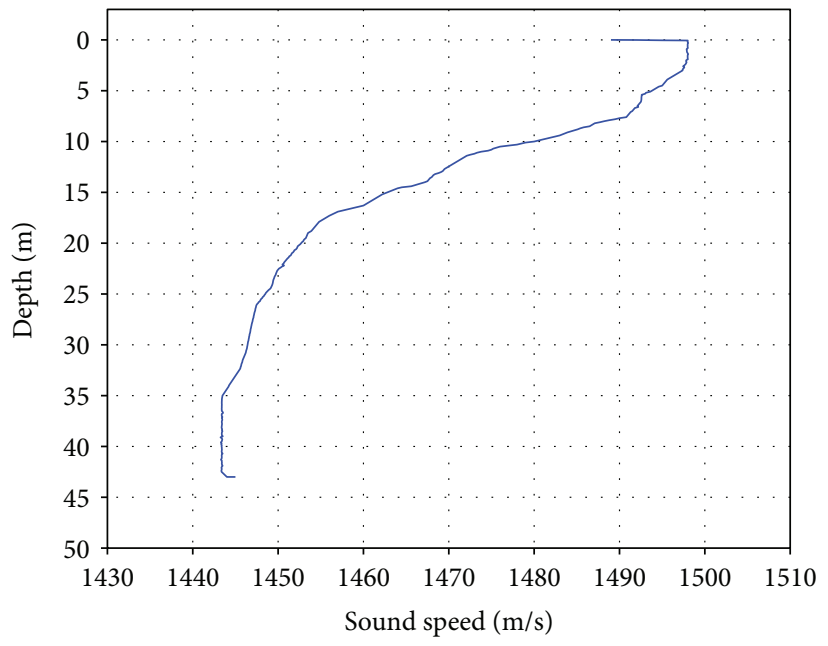

FIgURE 7: The sound speed profile. (Figure reproduced from Zhao et al. [17], under the Creative Commons Attribution License/ public domain).

TABLE 1: Experiment geographical environment parameters.

\begin{tabular}{lcc}
\hline Parameters & Acoustic source ship & Receiver ship \\
\hline GPS coordinates & $32^{\circ} 45.683^{\prime} \mathrm{N}$ & $32^{\circ} 42.272^{\prime} \mathrm{N}$ \\
Water depth $(\mathrm{m})$ & $111^{\circ} 33.807^{\prime} \mathrm{E}$ & $111^{\circ} 31.762^{\prime} \mathrm{E}$ \\
$D_{\text {eu }}{ }^{1}(\mathrm{~m})$ & 38 & 43 \\
${ }^{1} D_{\text {eu }}:$ depth of equipment underwater. & 20 & 25 \\
\hline
\end{tabular}



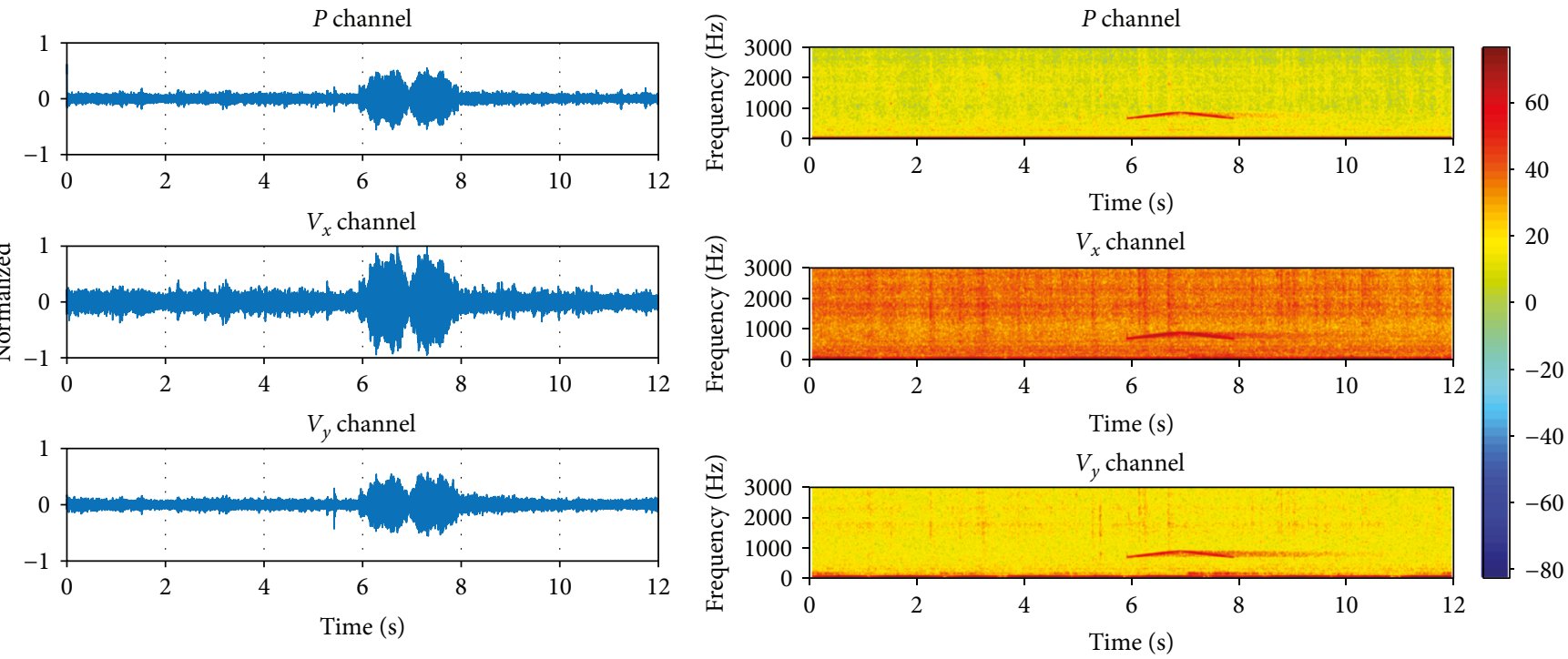

(b)

(a)

(b) The corresponding spectrograms.

The solution to this optimization problem is generally referred to as minimum variance distortionless response (MVDR) beamformer. The weighted vector of MVDR beamformer is easily derived [20]:

$$
\mathbf{w}=\frac{\mathbf{R}^{-1} \mathbf{a}_{s}(\theta)}{\mathbf{a}_{s}^{H}(\theta) \mathbf{R}^{-1} \mathbf{a}_{s}(\theta)} .
$$

Then, the spatial spectra are estimated by

$$
\widehat{P}(\theta)=\mathbf{w}^{H} \mathbf{R} \mathbf{w}=\frac{1}{\mathbf{a}_{s}^{H}(\theta) \mathbf{R}^{-1} \mathbf{a}_{s}(\theta)} .
$$

In practice, the matrix $\mathbf{R}$ is replaced by an estimate matrix $\widehat{\mathbf{R}}$. The same as the AVS CBF azimuth angle estimation method, the position corresponding to maximum value of $\widehat{P}(\theta)$ is the estimated source azimuth angle. The estimation procedure can be illustrated by Figure 3 as well.

2.2.4. AVS Music. AVS MUSIC azimuth spectrum estimation is realized by multiple signal classification technique based on exploiting the eigenstructure of the input covariance matrix. The covariance matrix can be decomposed into

$$
\mathbf{R}=\sum_{i=1}^{3} \lambda_{i} \boldsymbol{\Phi}_{i} \boldsymbol{\Phi}_{i}^{H}
$$

where $\lambda_{i}$ is the $i$ th eigenvalues and eigenvectors of the covariance matrix and $\boldsymbol{\Phi}_{i}$ is the corresponding $i$ th eigenvectors. We assume there is only one acoustic source, sorting the eigenvalue, that satisfies

$$
\lambda_{1} \geq \lambda_{2}=\lambda_{3}=\sigma^{2} .
$$

Equation (20) holds under the assumption of white noise, that is $E\left[\mathbf{n}(t) \mathbf{n}^{H}(t)\right]=\sigma^{2} \mathbf{I}$.
The signal subspace is composed of the first eigenvector. The noise subspace is composed of the rest two eigenvectors that uncorrelated with the signal eigenvector, as

$$
\begin{aligned}
\mathbf{U}_{s} & =\left[\boldsymbol{\Phi}_{1}\right], \\
\mathbf{U}_{n} & =\left[\boldsymbol{\Phi}_{2}, \boldsymbol{\Phi}_{3}\right] .
\end{aligned}
$$

Consequently, the corresponding spatial spectrum output is

$$
\widehat{P}(\theta)=\frac{1}{\mathbf{a}_{s}^{H}(\theta) \mathbf{U}_{n} \mathbf{U}_{n}^{H} \mathbf{a}_{s}(\theta)}=\frac{1}{\mathbf{a}_{s}^{H}(\theta)\left[\mathbf{I}-\mathbf{U}_{s} \mathbf{U}_{\mathbf{s}}^{\mathbf{H}}\right] \mathbf{a}_{s}(\theta)} .
$$

\section{Experiments and Results}

3.1. Experimental Setup. To evaluate and compare the practical application performance of the five investigated AVS azimuth angle estimation methods, we carried out serial open-lake experiments in Danjiangkou Reservoir from April to June 2016. The experimental setup is shown in Figure 4. The map of the site of these experiments is shown in Figure 5. As it shows, the reservoir is approximately $20 \mathrm{~km}$ long and $10 \mathrm{~km}$ wide. The bottom is muddy and sandy.

The two different ships are clearly identified as follows:

(a) Acoustic source ship, equipped with high-power transmitting acoustic source

(b) Receiver ship, equipped with AVSs and other related electronic devices

3.1.1. The AVS Introduction. A two-dimensional accelerometer structure AVS was applied in these open-lake experiments. It consists of two orthogonally oriented velocity hydrophones plus a pressure hydrophone, all colocated in space. Its directivity patterns (at $1000 \mathrm{~Hz}$ ) measured in the laboratory are shown in Figure 6(a). 

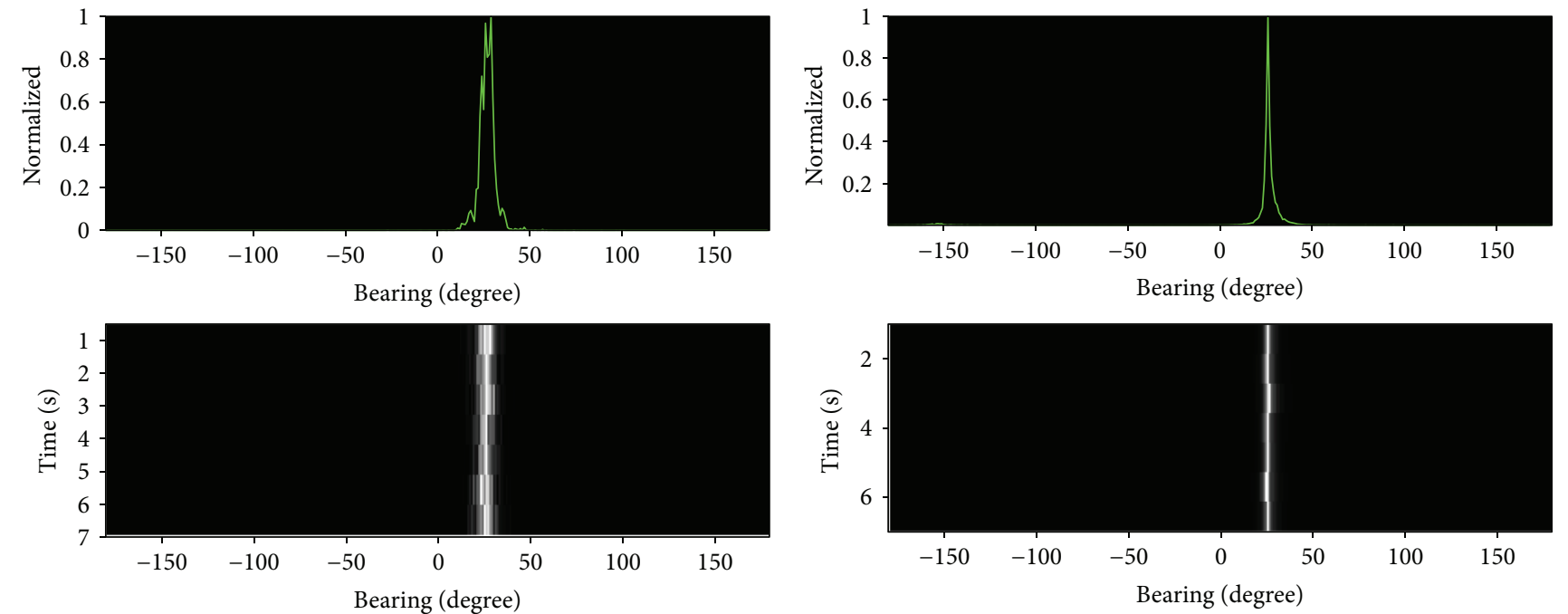

(a)

(b)
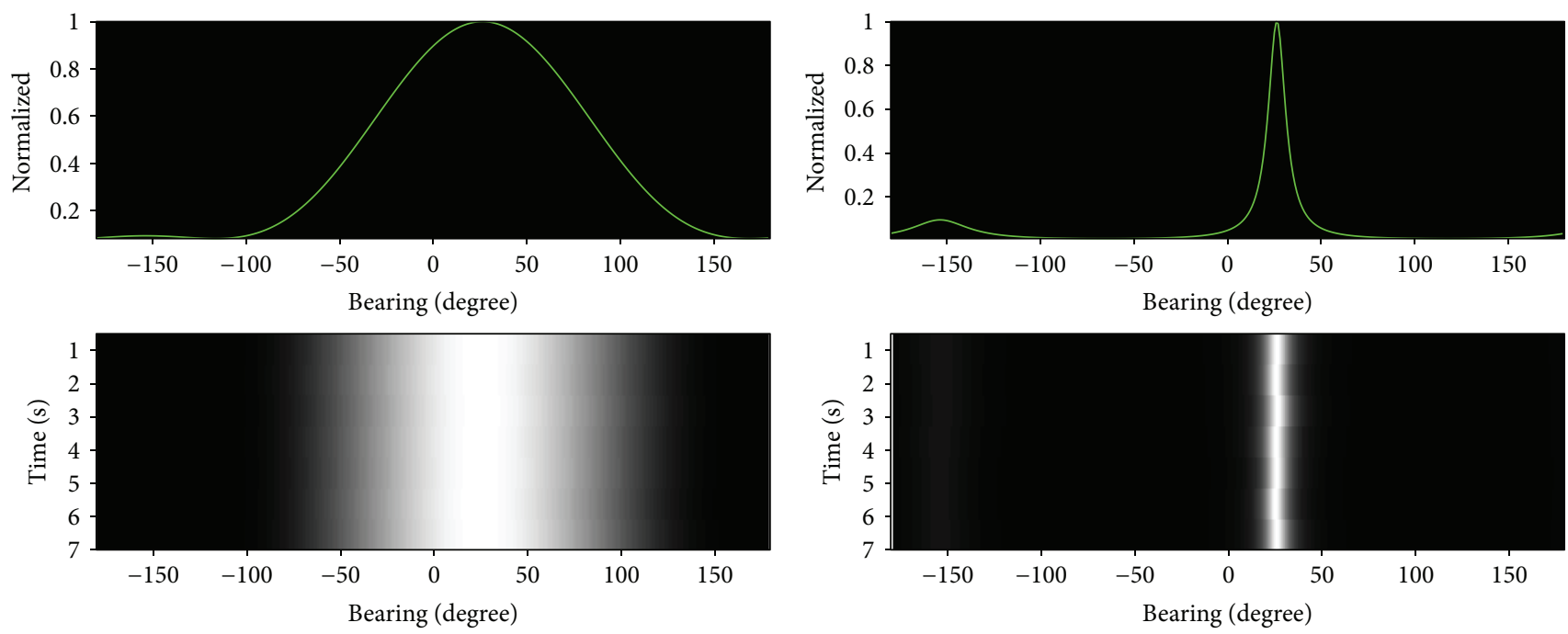

(c)

(d)
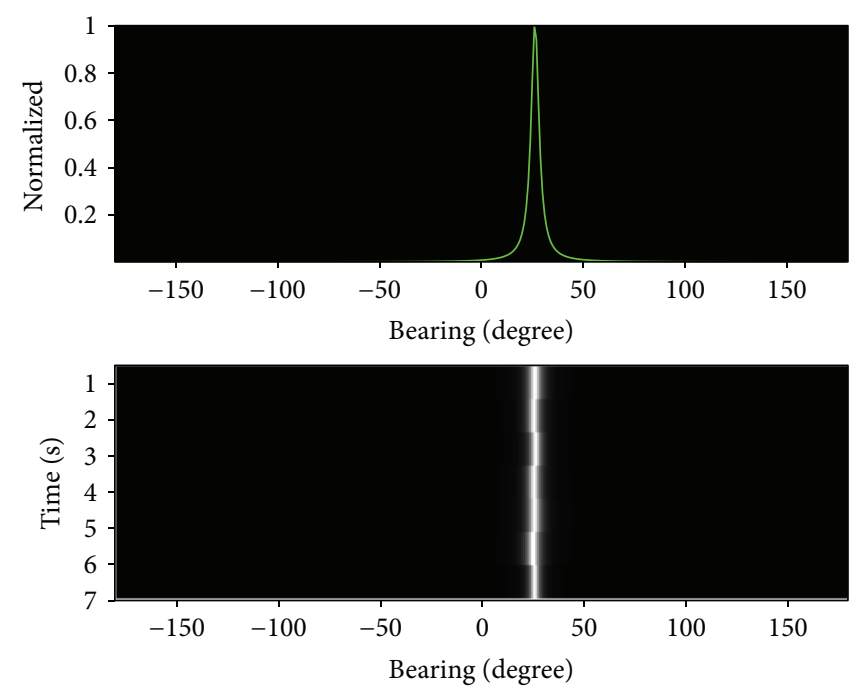

(e)

Figure 9: The real measurement data processing results: (a) CAIM; (b) WCAIM; (c) CBF; (d) MVDR; (e) MUSIC. 
TABLe 2: Analysis of the azimuth angle estimated results (true azimuth angle is $26.9^{\circ}$ ).

\begin{tabular}{|c|c|c|c|c|c|}
\hline S. number & $\operatorname{CAIM}\left({ }^{\circ}\right)$ & WCAIM $\left({ }^{\circ}\right)$ & $\mathrm{CBF}\left({ }^{\circ}\right)$ & $\operatorname{MVDR}\left({ }^{\circ}\right)$ & MUSIC $\left({ }^{\circ}\right)$ \\
\hline 1 & 26.1 & 26.1 & 26.1 & 26.1 & 26.1 \\
\hline 2 & 24.1 & 25.1 & 26.1 & 26.1 & 26.1 \\
\hline 3 & 26.6 & 25.6 & 25.6 & 25.6 & 25.6 \\
\hline 4 & 26.8 & 25.8 & 25.8 & 25.8 & 25.8 \\
\hline 5 & 26.6 & 26.6 & 26.6 & 26.6 & 26.6 \\
\hline 6 & 27.4 & 26.4 & 26.4 & 26.4 & 26.4 \\
\hline 7 & 28.7 & 25.7 & 25.7 & 25.7 & 25.7 \\
\hline Mean & 26.6 & 25.9 & 26.0 & 26.0 & 26.0 \\
\hline $\mathrm{RMSE}^{1}$ & 1.32 & 1.11 & 0.93 & 0.93 & 0.93 \\
\hline
\end{tabular}

${ }^{1}$ RMSE: root mean square error.
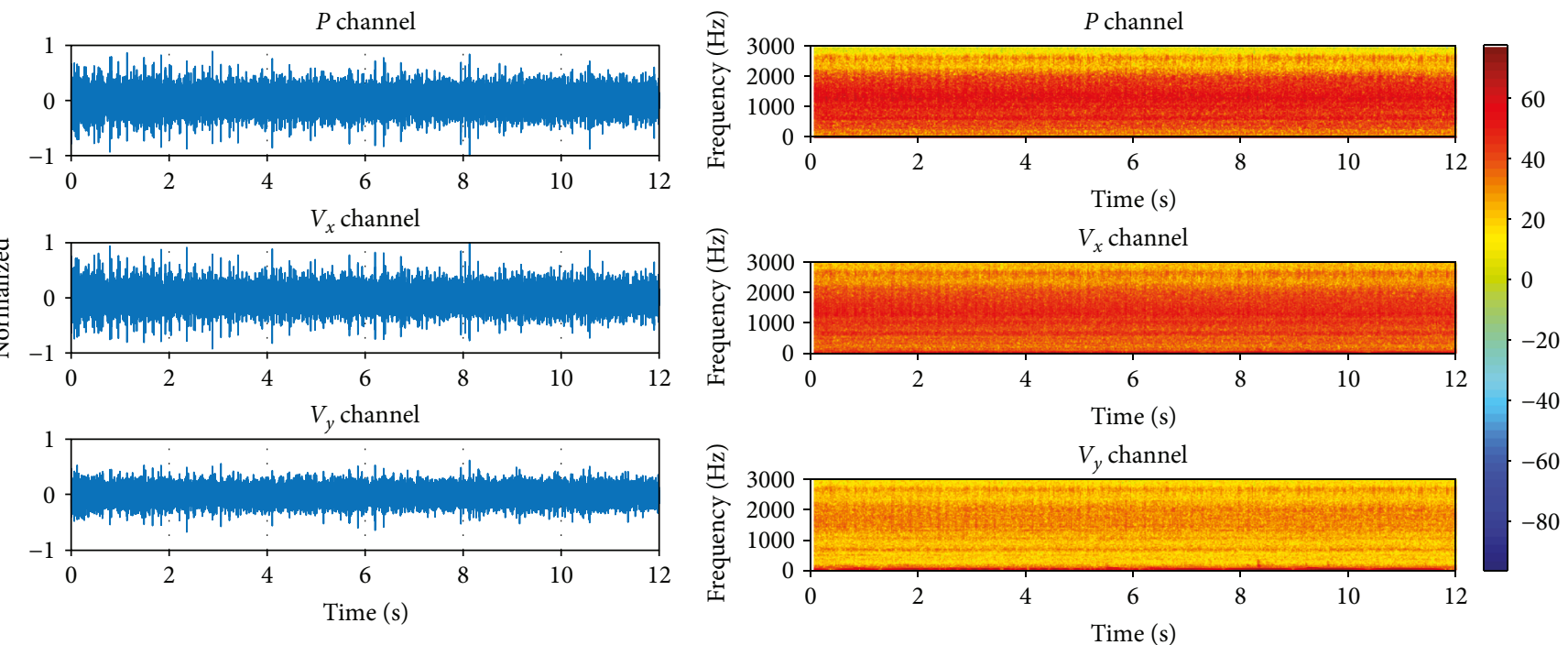

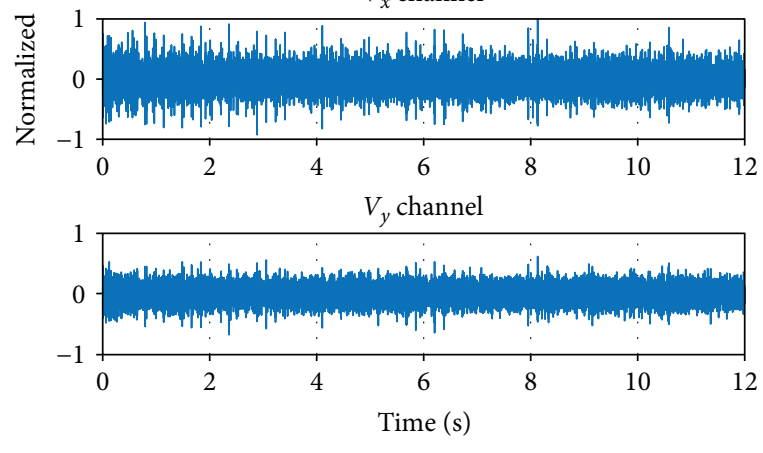

(a)

(b)

FIgURE 10: (a) The AVS output in time domain. (b) The corresponding spectrograms.

Consistent with theoretical measurement model, the sensitivity pattern of pressure is omnidirectional and that of velocity is dipole directional. A lateral rejection ratio of $38 \mathrm{~dB}$ or more against the other orthogonal axis is offered by the acceleration sensitivity on each axis. Meanwhile, as we can see in Figure 6(b), the maximum receiving sensitivity of the AVS between $200 \mathrm{~Hz}$ and $1500 \mathrm{~Hz}$ is $-188.8 \mathrm{~dB}$ re $1 \mu \mathrm{Pa}$ at $1 \mathrm{~m}$. In these experiments, the data from the AVS was acquired by the designated data acquisition unit.

3.1.2. Experiment Environment Parameters. Before the start of these lake experiments, the sound speed profile was measured by the CTD on the receiver ship. The sound speed profile of the site of these experiments is shown in Figure 7.

As shown in Figure 7, there is a serious negative sound speed gradient layer near the surface. The acoustic wave propagation is refracted downward in this layer. With increasing water depth, there exists an equal velocity profile layer below the depth of $35 \mathrm{~m}$. In accordance with underwater acoustic theory, this layer is relatively conducive to acoustic signal transmission. However, the AVS was down to about $25 \mathrm{~m}$ under the water surface since the bottom of the experiment site is an undulating area.

During the experiments, these two ships applied in the lake experiments were, respectively, anchored at a specific spot. The GPS coordinates of the two spots and the actual field-measured environment parameters are provided in detail in Table 1.

The actual distance and azimuth angle could be calculated according to the information of the GPS coordinates. The actual distance between the receiver ship and the acoustic source ship is $7.068 \mathrm{~km}$, and the actual azimuth angle between the connecting line and the geographic North Pole is $26.9^{\circ}$.

3.2. Results and Discussion. In these open-lake experiments, the acoustic source signal is a $650 \mathrm{~Hz}-850 \mathrm{~Hz}$ symmetrical linear frequency-modulated (SLFM) signal. The duration of 

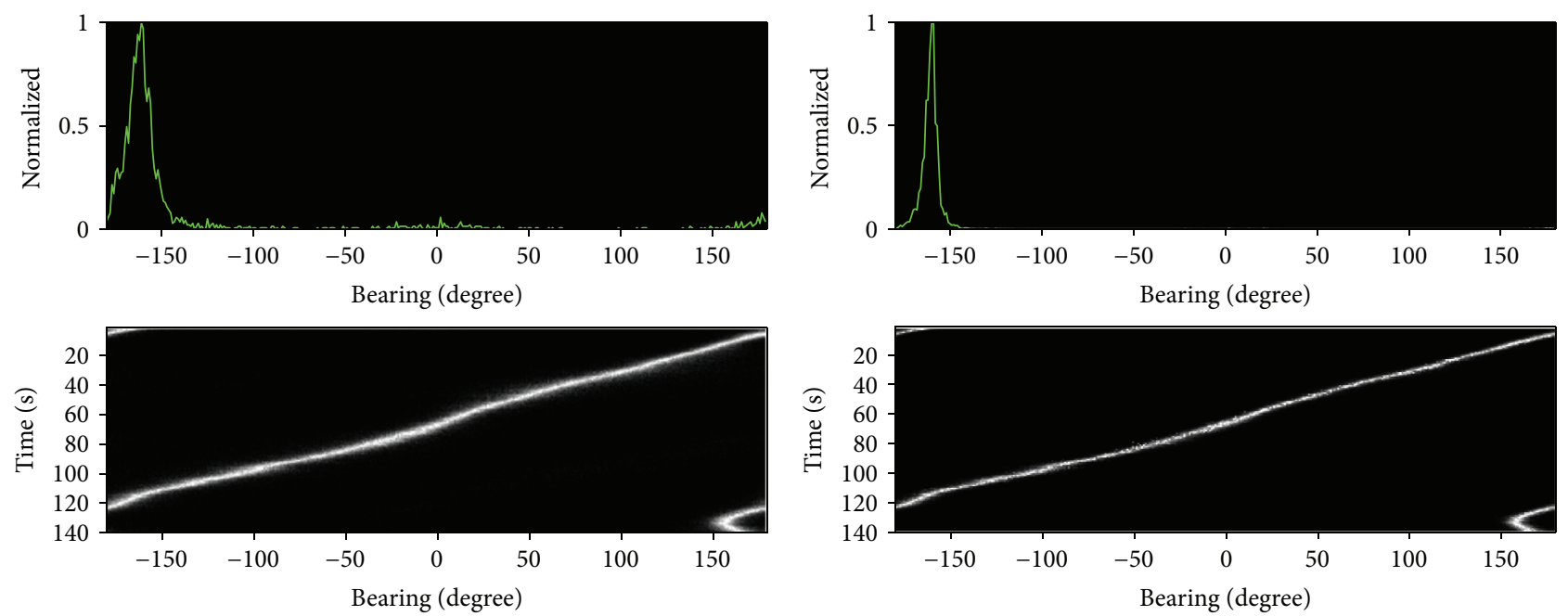

(a)

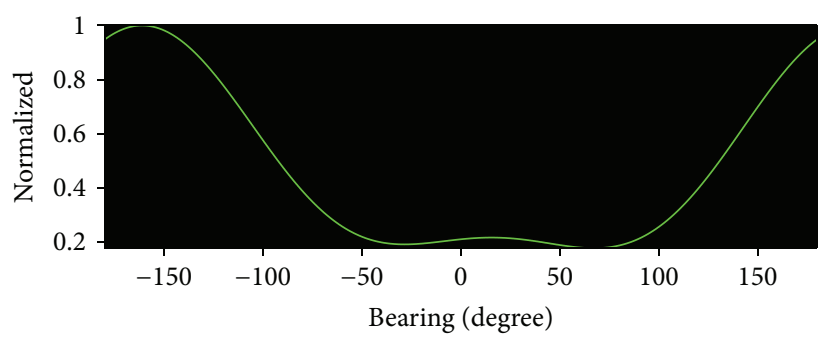

(b)
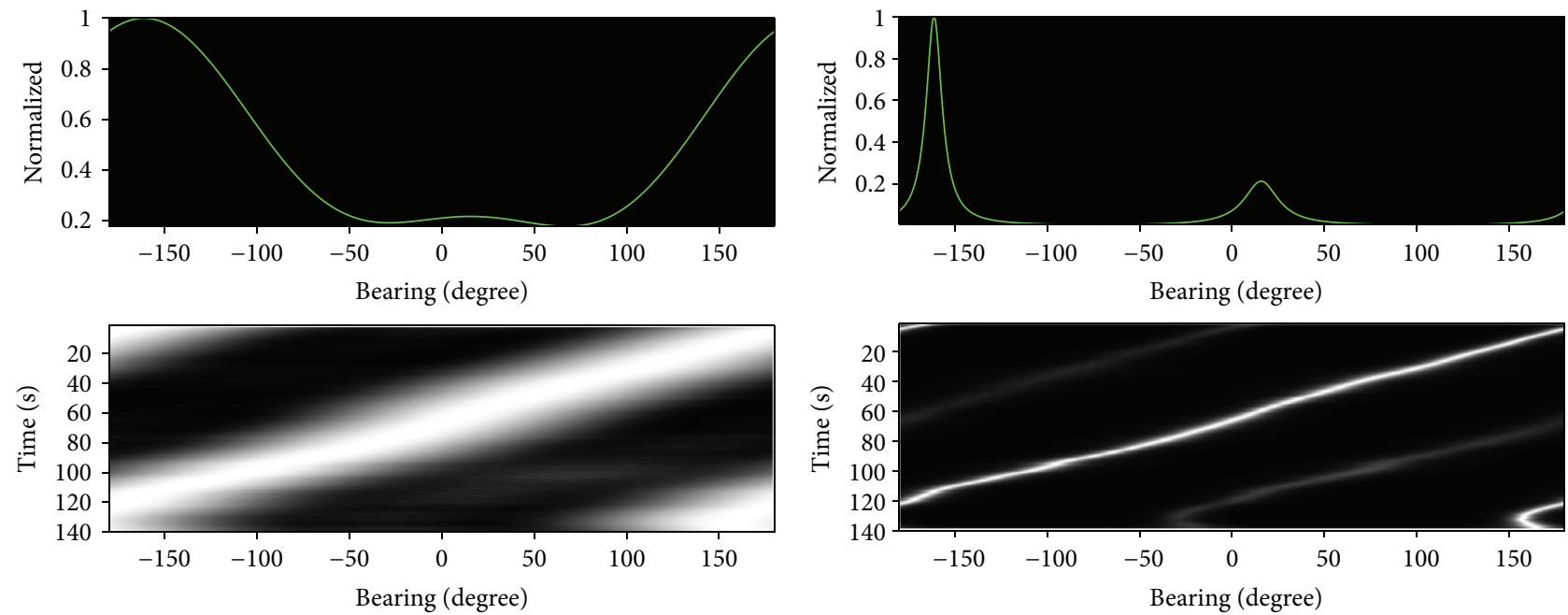

(c)

(d)
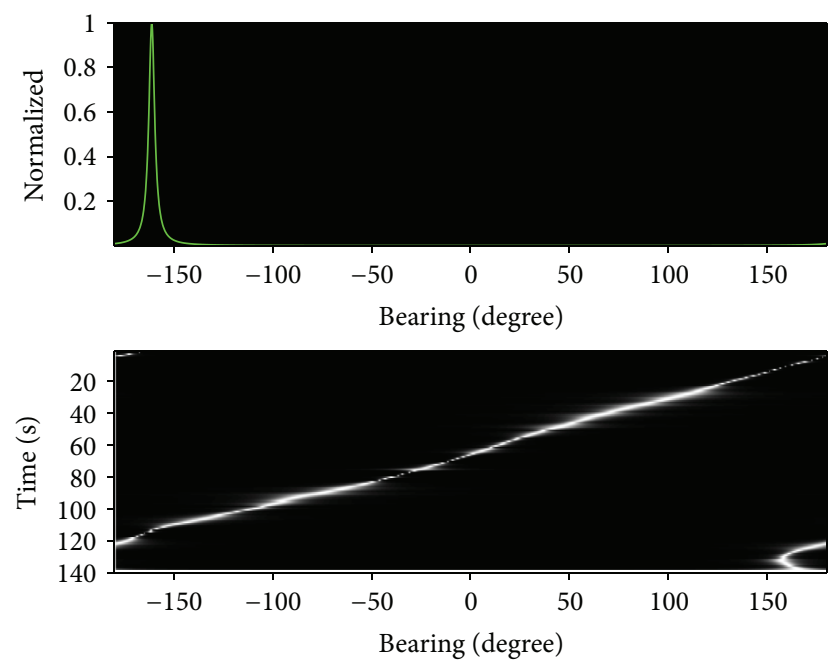

(e)

FIGURE 11: The real measurement data processing results: (a) CAIM; (b) WCAIM; (c) CBF; (d) MVDR; (e) MUSIC. 
the SLFM signal transmitted in these experiments is 2 seconds. The measured actual sound source level (SL) of the transmitted signal is $194.6 \mathrm{~dB}(1 \mathrm{re} \mu \mathrm{Pa}$ at $1 \mathrm{~m})$.

The multichannel output data in time domain from the AVS are shown in Figure 8(a). The variation in normalized amplitude of the different channel signals is clearly visible. Spectral domain analysis is further conducted and the corresponding spectrograms are shown in Figure 8(b). The color bar indicates the energy scale in this figure.

Then, the five methods introduced above are applied to estimate the source azimuth angle. The processing results of the lake experiment data are shown in Figure 9. The results are presented in two formats: (1) spatial spectrum and (2) bearing-time-record (BTR) plot. Both formats are very commonly used in passive sonar detection processing. The spatial spectrum presents spatial power over azimuth bearing only, while the BTR plot represents spectral power over both azimuth and time. The top row in each figure shows the directional spectra. BTR results are simultaneously shown in the bottom row.

For a more comprehensive comparison, the estimated results of seven times lake experiment data processed by these five investigated methods are listed in Table 2.

Comparing the results of the five methods in detail, we note that all the five methods investigated in this paper can effectively realize the azimuth angle estimation by using a single AVS. However, they are very different in terms of spatial resolution. CAIM, WCAIM, MVDR, and MUSIC methods perform much better than CBF method. The peaks of CAIM, WCAIM, MVDR, and MUSIC spatial spectrum are much sharper than that of CBF. As clearly shown in Figure 9(c), CBF suffers from low resolution and poor accuracy. The performance of WCAIM is very similar to that of MUSIC, or even slightly better. However, the computational complexity of WCAIM is much lower than that of the MUSIC. For WCAIM method, the azimuth angle is estimated by directly computing the acoustic intensity component of the different coordinate axis. But for the MUSIC method, the azimuth angle is estimated by computing the power spectra point by point in space range. The deficiency of MVDR is a weak "fake peak" emerging in the mirror image position.

Moreover, it is worth to point out that the impulse response is generated by multiple arrivals in such shallow water environment. The bias in these experimental results presented in Table 2 is due to the effect of multipath propagation.

In another representative experiment, a high-speed boat radiating mechanical noise is applied to make a further investigation of these five methods. The high-speed boat does circular motion around the receiver ship. Figure 10(a) shows the AVS multichannel output data in time domain. And the corresponding spectrograms are shown in Figure 10(b).

The frequency range of real measurement data processing is from $100 \mathrm{~Hz}$ to $2500 \mathrm{~Hz}$. The movement trajectory estimated results of the high-speed boat are shown in Figure 11.

These experimental results show that the movement trajectory of the high-speed boat is estimated accurately. The spectral resolution of the CBF method is much lower than that of the other four methods. It can be seen that the results estimated by the other four methods provide satisfactory information about the target bearing. As we expect, the same as the results shown in Figure 9, WCAIM and MUSIC both have the equally highest resolution; a weak "fake peak" exists in the mirror image position in the result estimated by MVDR. The MUSIC method requires prior knowledge of a number of sources to perform accurate subspace decomposition of the covariance matrix. The other methods do not require any prior information about the source. Hence, WCAIM method could achieve widespread use in practice, mainly because of its computation simplicity and its relative superior performance.

These experimental investigation results provide a valuable insight into the design and implementation of practical passive sonar detection systems. However, there are remaining research issues related to the azimuth estimation problem. For instance, it remains to be determined whether these methods can achieve good performance or not in a multitarget environment.

\section{Conclusions}

The AVSs are practical and versatile acoustic measurement sensors, with applications in underwater acoustic detection. We provided an experimental investigation of five popular azimuth angle estimation methods, CAIM, WCAIM, CBF, MVDR, and MUSIC, upon a single AVS configuration. According to the open-lake experiments and real measurement data processing results, the effectiveness of these methods is verified, and performances of these methods are compared in detail. All the five methods investigated in this paper can effectively realize the azimuth angle estimation by using only one single AVS. CAIM, WCAIM, MVDR, and MUSIC methods provide much superior performance in spatial resolution than CBF method. CBF suffers from relatively low resolution and poor accuracy. The performance of WCAIM is very similar to that of MUSIC, or even slightly better. Moreover, the computational complexity of WCAIM is much lower than that of the MUSIC. For WCAIM method, no prior information is required to estimate the azimuth angle. Therefore, among these methods, WCAIM method could achieve widespread use in practical engineering. The results presented in this paper reveal that AVS can be applied in a wider range of application in distributed underwater acoustic detection systems.

\section{Conflicts of Interest}

The authors declare no conflicts of interest.

\section{Acknowledgments}

This paper was funded by the China Scholarship Council (CSC), National Science Foundation of China (Grant no. 61371171 and 11374072), and Foundation of National Key Laboratory of Science and Technology on Underwater Acoustic Antagonizing. 


\section{References}

[1] M. K. Awad and K. T. Wong, "Recursive least-squares sourcetracking using one acoustic vector-sensor," IEEE Transactions on Aerospace and Electronic Systems, vol. 48, no. 4, pp. 30733083, 2012.

[2] J. Loo, J. Lloret, and J. H. Ortiz, Mobile Ad Hoc Networks: Current Status and Future Trends, CRC, Boca Raton, FL, USA, 2011.

[3] F. Niu, J. Hui, and Z. S. Zhao, "Research on vector acoustic focusing and shielding technology," Ocean Engineering, vol. 125, pp. 90-94, 2016.

[4] S. Sendra, J. Lloret, J. M. Jimenez, and L. Parra, "Underwater acoustic modems," IEEE Sensors Journal, vol. 16, no. 11, pp. 4063-4071, 2016.

[5] A. J. Poulsen, Robust vector sensor array processing and performance analysis, [Ph.D. dissertation], Massachusetts Institute of Technology, Boston, MA, USA, 2009.

[6] P. Tichavsky, K. T. Wong, and M. D. Zoltowski, "Near-field/ far-field azimuth and elevation angle estimation using a single vector-hydrophone," IEEE Transactions on Signal Processing, vol. 49, no. 11, pp. 2498-2510, 2001.

[7] P. Felisberto, O. Rodriguez, P. Santos, E. Ey, and S. M. Jesus, "Experimental results of underwater cooperative source localization using a single acoustic vector sensor," Sensors, vol. 13, no. 12, pp. 8856-8878, 2013.

[8] L. Zhang, D. Wu, X. Han, and Z. Zhu, "Feature extraction of underwater target signal using Mel frequency cepstrum coefficients based on acoustic vector sensor," Journal of Sensors, vol. 2016, Article ID 7864213, 11 pages, 2016.

[9] A. Song, A. Abdi, M. Badiey, and P. Hursky, "Experimental demonstration of underwater acoustic communication by vector sensors," IEEE Journal of Oceanic Engineering, vol. 36, no. 3, pp. 454-461, 2011.

[10] C. Wang, J. Yin, D. Huang, and A. Zielinski, "Experimental demonstration of differential OFDM underwater acoustic communication with acoustic vector sensor," Applied Acoustics, vol. 91, pp. 1-5, 2015.

[11] A. M. Thode, K. H. Kim, R. G. Norman, S. B. Blackwell, and C. R. Greene Jr., "Acoustic vector sensor beamforming reduces masking from underwater industrial noise during passive monitoring," The Journal of the Acoustical Society of America, vol. 139, no. 4, pp. EL105-EL111, 2016.

[12] P. Santos, O. C. Rodriguez, P. Felisberto, and S. M. Jesus, "Seabed geoacoustic characterization with a vector sensor array," The Journal of the Acoustical Society of America, vol. 128, no. 5, pp. 2652-2663, 2010.

[13] H.-W. Chen and J.-W. Zhao, "Wideband MVDR beamforming for acoustic vector sensor linear array," IEE Proceedings Radar, Sonar and Navigation, vol. 151, no. 3, pp. 158-162, 2004.

[14] K. Han and A. Nehorai, "Nested vector-sensor array processing via tensor modeling," IEEE Transactions on Signal Processing, vol. 62, no. 10, pp. 2542-2553, 2014.

[15] S. Najeem, K. Kiran, A. Malarkodi, and G. Latha, "Open lake experiment for direction of arrival estimation using acoustic vector sensor array," Applied Acoustics, vol. 119, pp. 94-100, 2017.

[16] G. L. D’Spain, J. C. Luby, G. R. Wilson, and R. A. Gramann, "Vector sensors and vector sensor line arrays: comments on optimal array gain and detection," The Journal of the Acoustical Society of America, vol. 120, no. 1, pp. 171-185, 2006.

[17] A. Zhao, L. Ma, X. Ma, and J. Hui, “An improved azimuth angle estimation method with a single acoustic vector sensor based on an active sonar detection system," Sensors, vol. 17, no. 12 , p. 412,2017

[18] A. Bereketli, M. B. Guldogan, T. Kolcak, T. Gudu, and A. L. Avsar, "Experimental results for direction of arrival estimation with a single acoustic vector sensor in shallow water," Journal of Sensors, vol. 2015, Article ID 401353, 10 pages, 2015.

[19] W. D. Zhang, L. G. Guan, G. J. Zhang, C. Y. Xue, K. R. Zhang, and J. P. Wang, "Research of DOA estimation based on single MEMS vector hydrophone," Sensors, vol. 9, no. 12, pp. 68236834, 2009.

[20] K. T. Wong and H. Chu, "Beam patterns of an underwater acoustic vector hydrophone located away from any reflecting boundary," IEEE Journal of Oceanic Engineering, vol. 27, no. 3, pp. 628-637, 2002.

[21] Y. I. Wu, S.-K. Lau, and K. T. Wong, "Near-field/far-field array manifold of an acoustic vector-sensor near a reflecting boundary," Journal of the Acoustical Society of America, vol. 139, no. 6, pp. 3159-3176, 2016.

[22] G. Liang, W. Ma, and Y. Wang, "Time-space transform: a novel signal processing approach for an acoustic vector-sensor," Science China Information Sciences, vol. 56, no. 4, pp. 1-11, 2013.

[23] P. K. Tam, K. T. Wong, and Y. Song, "An hybrid Cramér-Rao bound in closed form for direction-of-arrival estimation by an "acoustic vector sensor" with gain-phase uncertainties," IEEE Transactions on Signal Processing, vol. 62, no. 10, pp. 25042516, 2014. 


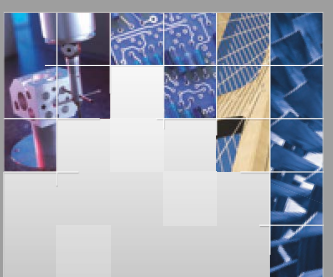

\section{Enfincering}
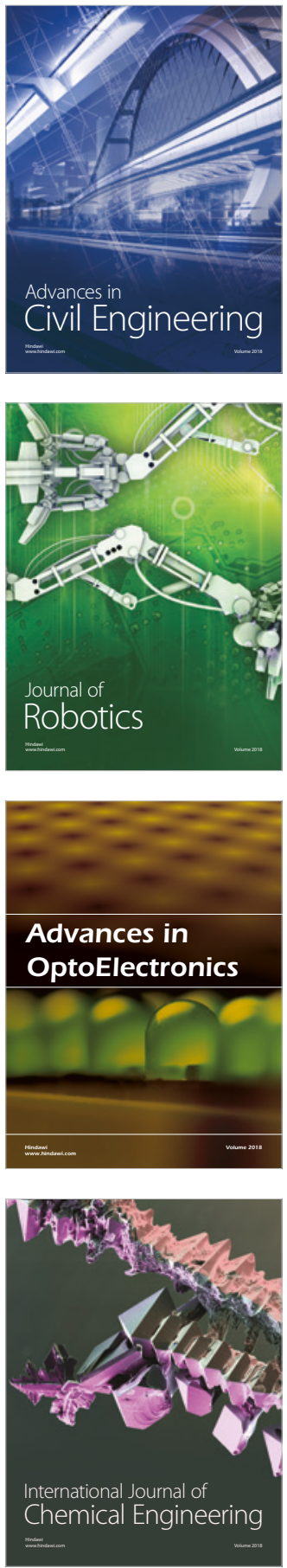

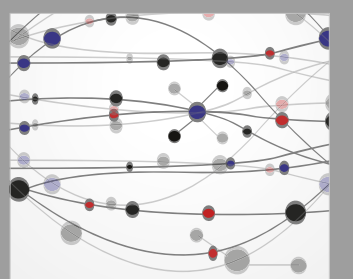

\section{Rotating \\ Machinery}

The Scientific World Journal

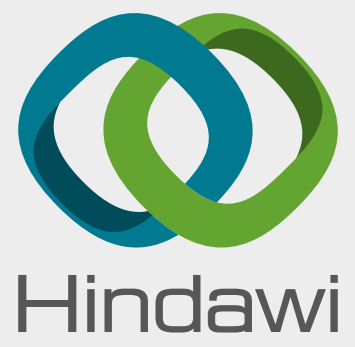

Submit your manuscripts at

www.hindawi.com
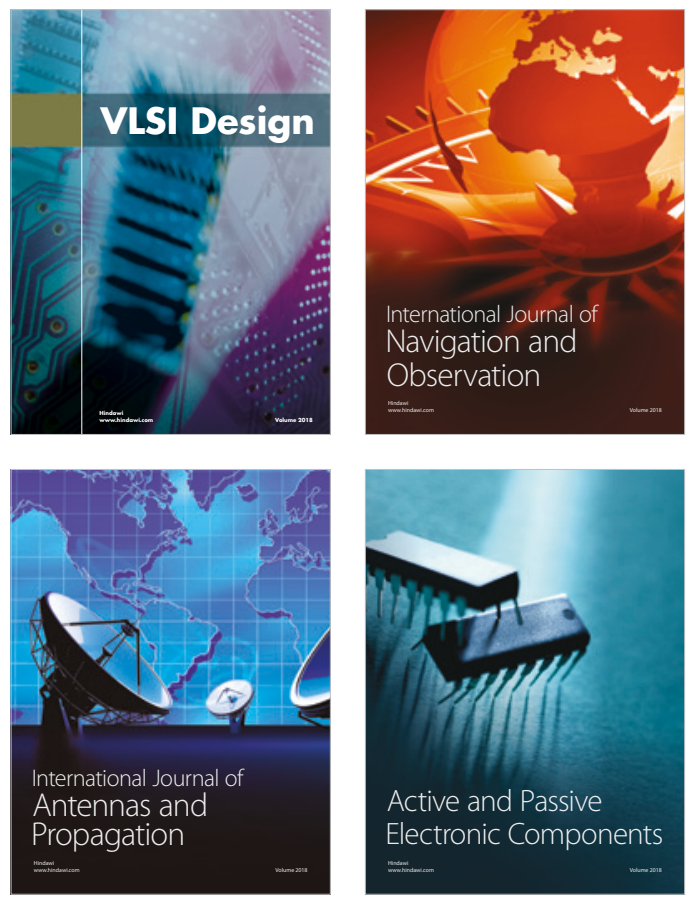
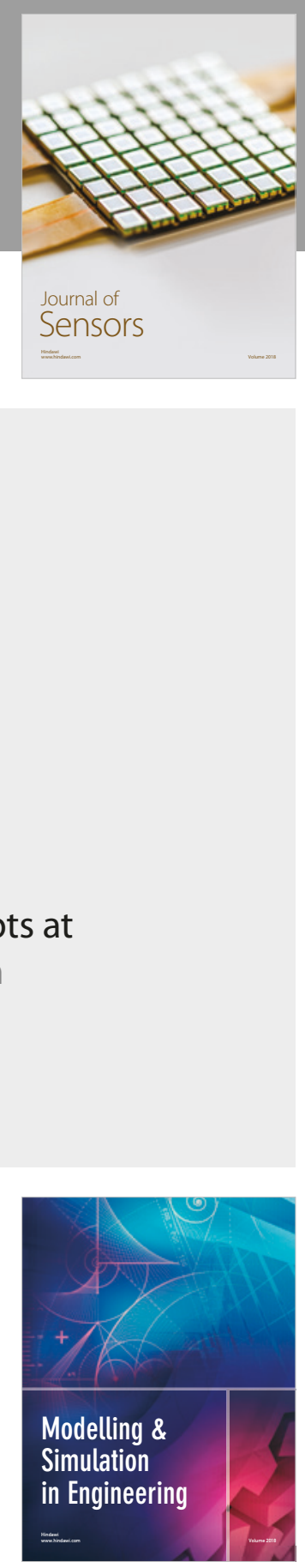

\section{Advances \\ Multimedia}
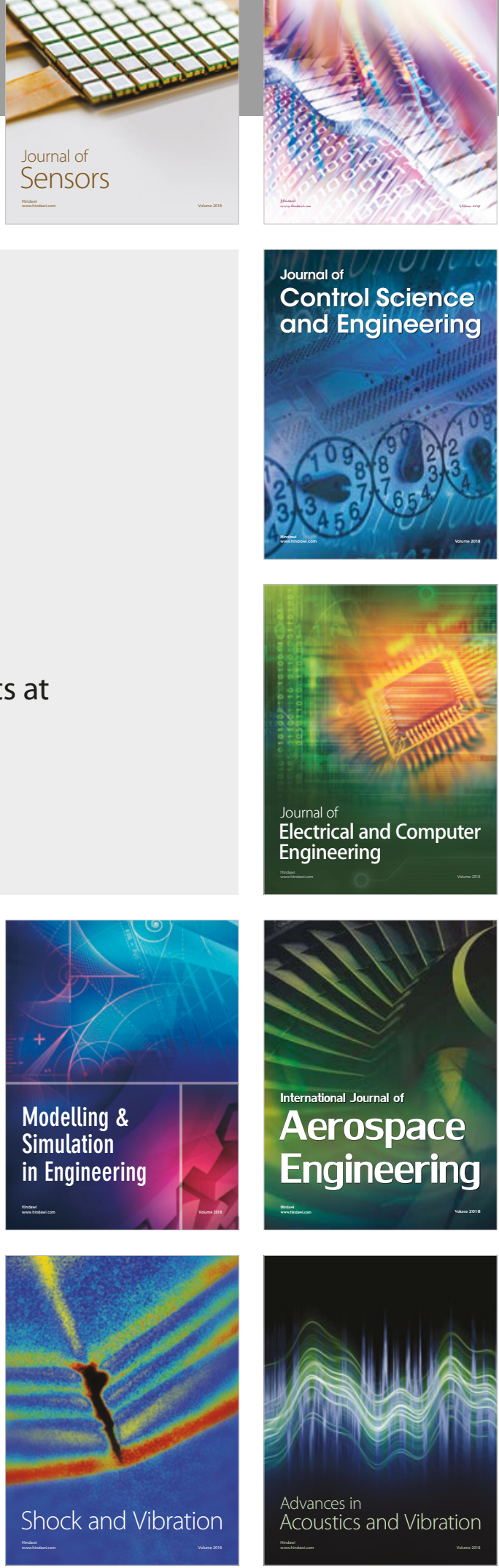